\title{
An ordinal multi-criteria decision-making procedure under imprecise linguistic assessments
}

\author{
José Luis GARCÍA-LAPRESTA* \\ PRESAD Research Group, BORDA Research Unit, IMUVA, Departamento de Economía \\ Aplicada, Universidad de Valladolid, Spain \\ Raquel GONZÁLEZ DEL POZO \\ PRESAD Research Group, IMUVA, Departamento de Economía Aplicada, Universidad de \\ Valladolid, Spain
}

\begin{abstract}
Many decision-making problems such as quality control analysis, market surveys or sensory analysis require ordered qualitative scales, rather than numerical ones. It is very common to assign some cardinal mathematical objects, such as numerical values, intervals of real numbers or fuzzy numbers, to the linguistic terms of ordered qualitative scales. However, when agents perceive that the psychological proximity between each pair of consecutive terms of the scale is not identical, these conversions are meaningless and an ordinal approach to deal with these non-uniform ordered qualitative scales is more appropriate. The aim of this paper is to introduce an ordinal multi-criteria decision-making procedure for ranking alternatives in the setting of ordered qualitative scales that are nonnecessarily uniform. The possibility of doubt is also considered, by allowing agents to assign two consecutive terms of the scale when they hesitate. The proposed procedure is applied to a real case study in which nine experts assessed eight wines regarding different criteria.
\end{abstract}

Keywords: Multiple criteria analysis; group decision-making; qualitative scales; ordinal proximity measures.

Email addresses: Corresponding author. lapresta@eco.uva.es (José Luis GARCÍA-LAPRESTA*), raquel.gonzalez.pozo@uva.es (Raquel GONZÁLEZ DEL POZO) 


\section{Introduction}

Decision-making problems are very common in operational research, management sciences, economics and engineering (e.g., quality control analysis, market surveys, sensory analysis, wine tasting, etc.).

A significant part of the problems addressed by decision-making can be considered as multi-criteria decision-making (MCDM) problems, where a group of experts assess alternatives regarding multiple criteria for obtaining the best alternative(s) or a ranking of them (see, for instance, Greco et al. [13] and Hong-Bin et al. [16]).

Many decision-making problems use ordered qualitative scales formed by linguistic terms, since words are more natural than numbers (see Zimmer [29], Teigen [24] and Windschitl and Wells [26], among others). The reason is that words are an appropriate tool for dealing with vagueness, imprecision and uncertainty in human decisions (see Larichev and Brown [19] and Fasolo and Bana e Costa [6], among others).

In general, ordered qualitative scales used in decision-making problems are Likert-type scales [20]. They are characterized by ordered response categories, a balanced number of positive and negative options and a numerical value assigned to each category. This is, for instance, the case of the scale \{'Objectionable', 'Poor', 'Deficient', 'Acceptable', 'Good', 'Excellent', 'Extraordinary'\}, used by the American Wine Society for evaluating some sensory aspects of wines, where each term is identified with a number: $0,1,2,3,4,5$ and 6 , respectively. However, when agents can perceive different proximities between the terms of the scale, this conversion of linguistic terms into numerical values is meaningless and could generate distinct outcomes when individual assessments are aggregated using different codifications of the same ordered qualitative scale (see, for instance, Roberts [23] and Franceschini et al. [7]).

Although in the literature there are few approaches dealing with non-uniform ordered qualitative scales in an ordinal way (see Franceschini et al. [7]), the vast majority of methods manage linguistic terms through cardinal approaches or fuzzy techniques (see Zadeh [28], Herrera-Viedma and López-Herrera [15] and Herrera et al. [14], among others). These cardinal approaches are practically equivalent to using numerical values and they make no sense in the context of non-uniform ordered qualitative scales.

On the other hand, the limited number of linguistic terms used in ordered qualitative scales, usually not beyond seven terms (see Miller [22], Kolesárová et 
al. [18] and Lozano et al. [21]), sometimes, it may lead agents to have difficulties for choosing a single linguistic term in their assessments. For this reason, it is interesting to allow agents to assign two (or more) consecutive terms of the scale when they are not confident about their opinions. This possibility of doubt is addressed by Travé-Massuyès and Piera [25] and Falcó et al. [5], among others, and it is also considered in this contribution.

The aim of this paper is to propose a MCDM procedure when agents evaluate a set of alternatives regarding different criteria by means of the linguistic terms of an ordered qualitative scale non-necessarily uniform. The procedure is developed in an ordinal way through the notion of ordinal proximity measure, introduced by García-Lapresta and Pérez-Román [10]. This proposal extends the procedures introduced by García-Lapresta and González del Pozo [8] and García-Lapresta and Pérez-Román [12].

The procedure follows a purely ordinal approach in all the steps. Given that the weights are numbers in the unit interval and they cannot multiply the linguistic assessments given by agents, we propose to replicate the assessments obtained for each alternative in each criterion a number of times following the proportions among weights. For instance, if there are three criteria with weights 0.2, 0.3 and 0.5, the corresponding assessments are replicated 2, 3 and 5 times, respectively.

Once the assessments are replicated, the procedure ranks the alternatives taking into account the medians of the ordinal proximities between the assessments given by the agents and the highest term of the scale. Since some alternatives can share the same medians, a tie-breaking procedure is provided. Additionally, the practicality of the proposed procedure is illustrated in a real case study in which nine experts assessed eight wines regarding different criteria.

Our procedure is related to the Majority Judgment voting system, introduced by Balinski and Laraki [1, 2], where voters evaluate candidates by means of linguistic assessments in an ordered qualitative scale, and candidates are ranked through the lower medians of the corresponding assessments. However, there are some differences between Majority Judgment and our approach.

1. Majority Judgment does not take into account whether the scales are uniform or not. If the scale is not uniform, using the (lower) median as overall assessment could not be representative of the individual assessments. In our procedure the ordinal degrees of proximity between the terms of the scale are crucial, and different ordinal proximity measures on the same 
scale can generate different rankings on the set of alternatives. Thus, our procedure is sensitive to the perception of the scale.

2. Selecting the lower median (of individual assessments, in Majority Judgment) could be considered as arbitrary when the number of assessments is low. In fact, if the upper median is considered, the outcome could be different. In our procedure the two medians (of degrees of proximity) are taken into account and we avoid loss of information.

3. Majority Judgment does not allow voters to hesitate between consecutive linguistic terms of the scale. Since usually qualitative scales have a low number of linguistic terms, it is common that voters hesitate. Thus, our procedure enhances the voter expressivity of Majority Judgment.

The rest of the paper is organized as follows. Section 2 is devoted to introduce ordinal proximity measures which allow us to work with non-uniform ordered qualitative scales in a purely ordinal way. In this section, we also address the possibility that agents assign two consecutive terms of an ordered qualitative scale to each alternative, when they are not confident about their opinions. To do that, we introduce an extension of ordinal proximity measures. Section 3 presents the proposed ordinal MCDM procedure. Section 4 includes the real case study. Finally, Section 5 concludes with some remarks.

\section{Measuring proximities between imprecise linguistic assessments}

In this section we introduce an ordinal procedure for measuring the proximities between imprecise linguistic assessments.

Along the paper we consider an ordered qualitative scale (OQS) $\mathcal{L}=$ $\left\{l_{1}, \ldots, l_{g}\right\}$, with $g \geq 3$ and $l_{1}<l_{2}<\cdots<l_{g}$. We say that $\mathcal{L}$ is uniform if the proximity between each pair of consecutive linguistic terms, $l_{r}$ and $l_{r+1}$ for $r \in\{1, \ldots, g-1\}$, is perceived as identical.

First we analyze how to measure the ordinal proximities between single linguistic terms of an OQS.

\subsection{Ordinal proximity measures}

In order to deal with non-uniform OQSs, we present the notion of ordinal proximity measure which was introduced by García-Lapresta and Pérez-Román [10].

An ordinal proximity measure is a mapping that assigns an ordinal degree of proximity to each pair of linguistic terms of an OQS $\mathcal{L}$. These ordinal degrees 
of proximity belong to a linear order $\Delta=\left\{\delta_{1}, \ldots, \delta_{h}\right\}$, with $\delta_{1} \succ \cdots \succ \delta_{h}$, being $\delta_{1}$ and $\delta_{h}$ the maximum and the minimum ordinal degrees of proximity, respectively.

Definition 1. ([10]) $A n$ ordinal proximity measure $(\mathbf{O P M})$ on $\mathcal{L}$ with values in $\Delta$ is a mapping $\pi: \mathcal{L}^{2} \longrightarrow \Delta$, where $\pi\left(l_{r}, l_{s}\right)=\pi_{r s}$ means the degree of proximity between $l_{r}$ and $l_{s}$, satisfying the following conditions:

1. Exhaustiveness: For every $\delta \in \Delta$, there exist $l_{r}, l_{s} \in \mathcal{L}$ such that $\delta=\pi_{r s}$.

2. Symmetry: $\pi_{s r}=\pi_{r s}$, for all $r, s \in\{1, \ldots, g\}$.

3. Maximum proximity: $\pi_{r s}=\delta_{1} \Leftrightarrow r=s$, for all $r, s \in\{1, \ldots, g\}$.

4. Monotonicity: $\pi_{r s} \succ \pi_{r t}$ and $\pi_{s t} \succ \pi_{r t}$, for all $r, s, t \in\{1, \ldots, g\}$ such that $r<s<t$.

Every OPM can be represented by a $g \times g$ symmetric matrix with coefficients in $\Delta$, where the elements in the main diagonal are $\pi_{r r}=\delta_{1}, r=1, \ldots, g$ :

$$
\left(\begin{array}{ccccc}
\pi_{11} & \cdots & \pi_{1 s} & \cdots & \pi_{1 g} \\
\cdots & \cdots & \cdots & \cdots & \cdots \\
\pi_{r 1} & \cdots & \pi_{r s} & \cdots & \pi_{r g} \\
\cdots & \cdots & \cdots & \cdots & \cdots \\
\pi_{g 1} & \cdots & \pi_{g s} & \cdots & \pi_{g g}
\end{array}\right) .
$$

This matrix is called proximity matrix associated with $\pi$.

A prominent class of OPMs, introduced by García-Lapresta et al. [9], is the one of metrizable OPMs which is based on linear metrics on OQSs.

Definition 2. ([9]) $A$ linear metric on $\mathcal{L}$ is a mapping $d: \mathcal{L}^{2} \longrightarrow \mathbb{R}$ satisfying the following conditions for all $r, s, t \in\{1, \ldots, g\}$ :

1. Positiveness: $d\left(l_{r}, l_{s}\right) \geq 0$.

2. Identity of indiscernibles: $d\left(l_{r}, l_{s}\right)=0 \Leftrightarrow l_{r}=l_{s}$.

3. Symmetry: $d\left(l_{s}, l_{r}\right)=d\left(l_{r}, l_{s}\right)$.

4. Linearity: $d\left(l_{r}, l_{t}\right)=d\left(l_{r}, l_{s}\right)+d\left(l_{s}, l_{t}\right)$ whenever $r<s<t$.

Definition 3. ([9]) $A n O P M \pi: \mathcal{L}^{2} \longrightarrow \Delta$ is metrizable if there exists a linear metric $d: \mathcal{L}^{2} \longrightarrow \mathbb{R}$ such that $\pi_{r s} \succ \pi_{t u} \Leftrightarrow d\left(l_{r}, l_{s}\right)<d\left(l_{t}, l_{u}\right)$, for all $r, s, t, u \in\{1, \ldots, g\}$.

\subsection{Ordering medians of ordinal degrees of proximity}

In the procedure introduced in Section 3 we will deal with medians of lists of ordinal degrees of proximity and we will need to compare medians. We now present a median operator in this context and an appropriate linear order on the set of feasible medians. 
Let $\boldsymbol{y}=\left(y_{1}, \ldots, y_{p}\right) \in \Delta^{p}$ be a vector of ordinal degrees of proximity whose components are ordered in a decreasing fashion, from the highest to the lowest degrees. If $p$ is even, then $\boldsymbol{y}$ has two medians, say $\delta_{r}, \delta_{s} \in \Delta$ such as $r \leq s$. However, if $p$ is odd, then $\boldsymbol{y}$ has a unique median, say $\delta_{r} \in \Delta$.

In order to unify the assignment of medians and to avoid loss of information, we would consider the pair of medians of ordinal degrees of proximity as follows:

1. If the number of ordinal degrees of proximity is even, then consider the two medians.

2. If the number of ordinal degrees of proximity is odd, then duplicate the median.

Formally, the median operator is the mapping

$$
M: \bigcup_{p=1}^{\infty} \Delta^{p} \longrightarrow \Delta_{2},
$$

that assigns the corresponding pair of medians to each vector of ordinal degrees of proximity, where $\Delta_{2}=\left\{\left(\delta_{r}, \delta_{s}\right) \in \Delta^{2} \mid r \leq s\right\}$ is the set of feasible medians.

Once the medians of ordinal degrees of proximity are obtained, it should be necessary to have an appropriate linear order on $\Delta_{2}$ to rank order the corresponding pairs of medians.

The binary relation $\succcurlyeq_{b}$ on $\Delta_{2}$ defined as

$$
\left(\delta_{r}, \delta_{s}\right) \succcurlyeq_{b}\left(\delta_{t}, \delta_{u}\right) \Leftrightarrow(r \leq t \text { and } s \leq u)
$$

for all $\left(\delta_{r}, \delta_{s}\right),\left(\delta_{t}, \delta_{u}\right) \in \Delta_{2}$, is a partial order (reflexive, antisymmetric and transitive). We say that $\succcurlyeq_{b}$ is the basic order on $\Delta_{2}$. The Hasse diagram of $\succcurlyeq_{b}$ for $h=4$ is shown in Fig. 1 .

Every admissible weak or linear order on $\Delta_{2}$ should be an extension ${ }^{1}$ of $\succcurlyeq_{b}$.

We now present a suitable linear order $\succeq_{2}$ on $\Delta_{2}$ introduced by GarcíaLapresta and Pérez-Román [11]. We note that it is related to the one given by

\footnotetext{
${ }^{1} \mathrm{~A}$ weak or linear order $\succcurlyeq$ on $\Delta_{2}$ extends $\succcurlyeq_{b}$ if $\left(\delta_{r}, \delta_{s}\right) \succcurlyeq_{b}\left(\delta_{t}, \delta_{u}\right) \Rightarrow\left(\delta_{r}, \delta_{s}\right) \succcurlyeq\left(\delta_{t}, \delta_{u}\right)$, for all $\left(\delta_{r}, \delta_{s}\right),\left(\delta_{t}, \delta_{u}\right) \in \Delta_{2}$.
} 


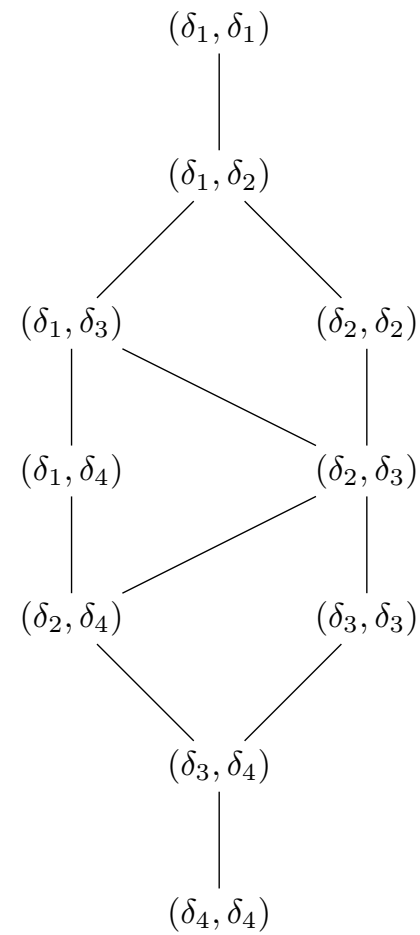

Figure 1: The Hasse diagram of $\succcurlyeq_{b}$ for $h=4$.

$\mathrm{Xu}$ and Yager [27] in the setting of intervals of real numbers ${ }^{2}$. It is defined as

$$
\left(\delta_{r}, \delta_{s}\right) \succeq_{2}\left(\delta_{t}, \delta_{u}\right) \Leftrightarrow\left\{\begin{array}{l}
r+s<t+u \\
\text { or } \\
r+s=t+u \text { and } s-r \leq u-t,
\end{array}\right.
$$

for all $\left(\delta_{r}, \delta_{s}\right),\left(\delta_{t}, \delta_{u}\right) \in \Delta_{2}$.

It is easy to check that if $r+s=t+u$, then $s-r \leq u-t \Leftrightarrow r \geq t \Leftrightarrow s \leq u$. Obviously, $\left(\delta_{r}, \delta_{r}\right) \succeq\left(\delta_{t}, \delta_{t}\right) \Leftrightarrow r \leq t$.

\footnotetext{
${ }^{2}$ As mentioned by Bustince et al. [4], it corresponds to the lexicographic order of random variables, where the expected value is the primary criterion, and the variance is the second one.
} 
For instance, for $h=4, \succeq_{2}$ extends $\succcurlyeq_{b}$ (see Fig. 1) in the following way:

$$
\begin{array}{r}
\left(\delta_{1}, \delta_{1}\right) \succ_{2}\left(\delta_{1}, \delta_{2}\right) \succ_{2}\left(\delta_{2}, \delta_{2}\right) \succ_{2}\left(\delta_{1}, \delta_{3}\right) \succ_{2}\left(\delta_{2}, \delta_{3}\right) \succ_{2} \\
\left(\delta_{1}, \delta_{4}\right) \succ_{2}\left(\delta_{3}, \delta_{3}\right) \succ_{2}\left(\delta_{2}, \delta_{4}\right) \succ_{2}\left(\delta_{3}, \delta_{4}\right) \succ_{2}\left(\delta_{4}, \delta_{4}\right) .
\end{array}
$$

It is important to mention that $\succeq_{2}$ is not the only possible extension of $\succcurlyeq_{b}$ and other linear orders on $\Delta_{2}$ can be considered (see, for instance, Bustince et al. [4]).

\subsection{Extension of OPMs}

Since sometimes agents may hesitate between two consecutive terms of the scale when they provide their assessments over a set of alternatives (see GarcíaLapresta and González del Pozo [8]), we will allow agents to assign two consecutive linguistic terms of the OQS to each alternative when they are not confident about their opinions. The set of these intervals is denoted by

$$
\mathcal{L}_{2}=\left\{\left[l_{r}, l_{s}\right] \mid r, s \in\{1, \ldots, g\}, s \in\{r, r+1\}\right\} .
$$

The elements of $\mathcal{L}_{2}$ are either subsets of two consecutive linguistic terms, $\left[l_{r}, l_{r+1}\right]=\left\{l_{r}, l_{r+1}\right\}$, or a single linguistic term, $\left[l_{r}, l_{r}\right]=\left\{l_{r}\right\}$. For practical reasons we identify $\left[l_{r}, l_{r}\right]=\left\{l_{r}\right\}$ with $l_{r}$. Notice that the cardinality of $\mathcal{L}_{2}$ is $2 g-1$.

We now extend the original linear order $<$ on $\mathcal{L}$ to $\mathcal{L}_{2}$ in the natural way: $l_{r}<\left[l_{r}, l_{r+1}\right]<l_{r+1}$, for every $r \in\{1, \ldots, g-1\}$, and generalize the notion of OPM to the setting of $\mathcal{L}_{2}$.

Definition 4. Given an $O P M \pi: \mathcal{L}^{2} \longrightarrow \Delta$, the extension of $\pi$ to $\mathcal{L}_{2}$ is the mapping $\pi^{*}:\left(\mathcal{L}_{2}\right)^{2} \longrightarrow \Delta_{2}$ defined as

$$
\pi^{*}\left(\left[l_{r}, l_{s}\right],\left[l_{t}, l_{u}\right]\right)= \begin{cases}M\left(\pi_{r t}, \pi_{r u}, \pi_{s t}, \pi_{s u}\right), & \text { if }\left[l_{r}, l_{s}\right] \neq\left[l_{t}, l_{u}\right], \\ \left(\delta_{1}, \delta_{1}\right), & \text { if }\left[l_{r}, l_{s}\right]=\left[l_{t}, l_{u}\right] .\end{cases}
$$

The pairs of ordinal degrees of proximity $\pi^{*}\left(\left[l_{r}, l_{s}\right],\left[l_{t}, l_{u}\right]\right) \in \Delta_{2}$ will be arranged in a $(2 g-1) \times(2 g-1)$ symmetric matrix whose elements in the main diagonal correspond to the maximum proximity, $\left(\delta_{1}, \delta_{1}\right)$. This matrix is called proximity matrix associated with $\pi^{*}$.

Remark 1. Given an OPM $\pi: \mathcal{L}^{2} \longrightarrow \Delta$, the extension of $\pi$ to $\mathcal{L}_{2}$ satisfies the following properties for all $\left[l_{r}, l_{s}\right],\left[l_{t}, l_{u}\right],\left[l_{v}, l_{w}\right] \in \mathcal{L}_{2}$ : 
1. Symmetry: $\pi^{*}\left(\left[l_{r}, l_{s}\right],\left[l_{t}, l_{u}\right]\right)=\pi^{*}\left(\left[l_{t}, l_{u}\right],\left[l_{r}, l_{s}\right]\right)$.

2. Maximum proximity: $\pi^{*}\left(\left[l_{r}, l_{s}\right],\left[l_{t}, l_{u}\right]\right)=\left(\delta_{1}, \delta_{1}\right) \Leftrightarrow\left[l_{r}, l_{s}\right]=\left[l_{t}, l_{u}\right]$.

3. Monotonicity: If $\left[l_{r}, l_{s}\right]<\left[l_{t}, l_{u}\right]<\left[l_{v}, l_{w}\right]$, then $\pi^{*}\left(\left[l_{r}, l_{s}\right],\left[l_{t}, l_{u}\right]\right) \succ_{2}$ $\pi^{*}\left(\left[l_{r}, l_{s}\right],\left[l_{v}, l_{w}\right]\right)$ and $\pi^{*}\left(\left[l_{t}, l_{u}\right],\left[l_{v}, l_{w}\right]\right) \succ_{2} \pi^{*}\left(\left[l_{r}, l_{s}\right],\left[l_{v}, l_{w}\right]\right)$.

Example 1. Consider the OQS $\mathcal{L}=\left\{l_{1}, l_{2}, l_{3}\right\}$ equipped with the metrizable OPM $\pi: \mathcal{L} \longrightarrow \Delta=\left\{\delta_{1}, \delta_{2}, \delta_{3}, \delta_{4}\right\}$ with associated proximity matrix ${ }^{3}$

$$
A_{23}=\left(\begin{array}{ccc}
\delta_{1} & \delta_{2} & \delta_{4} \\
& \delta_{1} & \delta_{3} \\
& & \delta_{1}
\end{array}\right)
$$

Then, $\mathcal{L}_{2}=\left\{l_{1},\left[l_{1}, l_{2}\right], l_{2},\left[l_{2}, l_{3}\right], l_{3}\right\}$ and $\Delta_{2}=\left\{\left(\delta_{1}, \delta_{1}\right),\left(\delta_{1}, \delta_{2}\right),\left(\delta_{1}, \delta_{3}\right),\left(\delta_{1}, \delta_{4}\right),\left(\delta_{2}, \delta_{2}\right),\left(\delta_{2}, \delta_{3}\right),\left(\delta_{2}, \delta_{4}\right),\left(\delta_{3}, \delta_{3}\right),\left(\delta_{3}, \delta_{4}\right),\left(\delta_{4}, \delta_{4}\right)\right\}$.

For instance,

$$
\pi^{*}\left(\left[l_{1}, l_{2}\right], l_{3}\right)=M\left(\pi_{13}, \pi_{13}, \pi_{23}, \pi_{23}\right)=M\left(\delta_{4}, \delta_{4}, \delta_{3}, \delta_{3},\right)=\left(\delta_{3}, \delta_{4}\right) .
$$

After some computations, we obtain the proximity matrix associated with $\pi^{*}:$

$$
\begin{gathered}
\left(\begin{array}{ccccc}
\pi^{*}\left(l_{1}, l_{1}\right) & \pi^{*}\left(l_{1},\left[l_{1}, l_{2}\right]\right) & \pi^{*}\left(l_{1}, l_{2}\right) & \pi^{*}\left(l_{1},\left[l_{2}, l_{3}\right]\right) & \pi^{*}\left(l_{1}, l_{3}\right) \\
& \pi^{*}\left(\left[l_{1}, l_{2}\right],\left[l_{1}, l_{2}\right]\right) & \pi^{*}\left(\left[l_{1}, l_{2}\right], l_{2}\right) & \pi^{*}\left(\left[l_{1}, l_{2}\right],\left[l_{2}, l_{3}\right]\right) & \pi^{*}\left(\left[l_{1}, l_{2}\right], l_{3}\right) \\
& \pi^{*}\left(l_{2}, l_{2}\right) & \pi^{*}\left(l_{2},\left[l_{2}, l_{3}\right]\right) & \pi^{*}\left(l_{2}, l_{3}\right) \\
& & & \pi^{*}\left(\left[l_{2}, l_{3}\right],\left[l_{2}, l_{3}\right]\right) & \pi^{*}\left(\left[l_{2}, l_{3}\right], l_{3}\right) \\
& & & \pi^{*}\left(l_{3}, l_{3}\right)
\end{array}\right)= \\
\left(\begin{array}{ccccc}
\left(\delta_{1}, \delta_{1}\right) & \left(\delta_{1}, \delta_{2}\right) & \left(\delta_{2}, \delta_{2}\right) & \left(\delta_{2}, \delta_{4}\right) & \left(\delta_{4}, \delta_{4}\right) \\
& \left(\delta_{1}, \delta_{1}\right) & \left(\delta_{1}, \delta_{2}\right) & \left(\delta_{2}, \delta_{3}\right) & \left(\delta_{3}, \delta_{4}\right) \\
& \left(\delta_{1}, \delta_{1}\right) & \left(\delta_{1}, \delta_{3}\right) & \left(\delta_{3}, \delta_{3}\right) \\
& & \left(\delta_{1}, \delta_{1}\right) & \left(\delta_{1}, \delta_{3}\right) \\
& & & \left(\delta_{1}, \delta_{1}\right)
\end{array}\right) .
\end{gathered}
$$
$\pi^{*}$.

Notice that $\pi^{*}$ is not exhaustive: $\left(\delta_{1}, \delta_{4}\right)$ does not belong to the image of

\footnotetext{
${ }^{3}$ The subindices 23 of the matrix $A_{23}$ correspond to the subindices of the $\delta$ 's appearing in the coefficients just over the main diagonal. We will follow the same pattern in subsequent matrices.
} 


\section{The ordinal MCDM procedure}

Many MCDM problems use linguistic terms for assessing and ranking a set of alternatives.

Balinski and Laraki $[2,21.3]$ introduced an extension of their Majority Judgment voting system to multicritieria problems. In their procedure, alternatives are evaluated regarding several criteria by means of an OQS. Weights are attached to each criterion according to its importance, and the linguistics assessments obtained for each alternative are replicated according to the weights of the corresponding criteria. Once the assessments are replicated, the alternatives are ranked by using the Majority Judgment voting system.

However, the possibility that agents may perceive different proximities between the linguistic terms of an OQS is not addressed in the Multicriteria Majority Judgment proposed by Balinski and Laraki $[2,21.3]$.

In this section, we present a MCDM procedure for ranking a set of alternatives regarding different criteria assessed through an OQS, considering the non-uniformity of the scale and the possibility that agents can select two consecutive terms of the scale when they hesitate ${ }^{4}$. The proposed procedure is an extension to one introduced recently by García-Lapresta and Pérez-Román [12] in a more simple context.

Let $A=\{1, \ldots, m\}$, with $m \geq 2$, be a set of agents and let $X=\left\{x_{1}, \ldots, x_{n}\right\}$, with $n \geq 2$, be the set of alternatives which have to be evaluated by the agents regarding a set of different criteria $C=\left\{c_{1}, \ldots, c_{q}\right\}$ through the intervals of linguistic terms of $\mathcal{L}_{2}$. Consider that $\mathcal{L}$ is equipped with an OPM $\pi: \mathcal{L}^{2} \longrightarrow \Delta$. We also consider the extension of $\pi$ to $\mathcal{L}_{2}, \pi^{*}:\left(\mathcal{L}_{2}\right)^{2} \longrightarrow \Delta_{2}$.

The opinions of all the agents over all the alternatives regarding the criterion $c_{k} \in C$ are collected in a profile $V^{k}$, that is a matrix of $m$ rows and $n$ columns with coefficients in $\mathcal{L}_{2}$ :

$$
V^{k}=\left(\begin{array}{ccccc}
v_{1}^{1, k} & \cdots & v_{i}^{1, k} & \cdots & v_{n}^{1, k} \\
\cdots & \cdots & \cdots & \cdots & \cdots \\
v_{1}^{a, k} & \cdots & v_{i}^{a, k} & \cdots & v_{n}^{a, k} \\
\cdots & \cdots & \cdots & \cdots & \cdots \\
v_{1}^{m, k} & \cdots & v_{i}^{m, k} & \cdots & v_{n}^{m, k}
\end{array}\right),
$$

\footnotetext{
${ }^{4}$ Notice that this is technically equivalent to adding new linguistic terms, but without introducing explicitly new words.
} 
where $v_{i}^{a, k}$ is the assessment given by the agent $a$ to the alternative $x_{i}$ with respect to the criterion $c_{k}$.

Since each criterion may have different importance in the decision, we consider a weighting vector $\left(w_{1}, \ldots, w_{q}\right) \in[0,1]^{q}$, with $w_{1}+\cdots+w_{q}=1$. For practical reasons, we assume that these weights have at most two decimals, i.e., the percentages $100 \cdot w_{1}, \ldots, 100 \cdot w_{q}$ are integer numbers.

To rank the alternatives, the procedure is divided in the following steps:

- Step 1. Gather the assessments given by the agents in the corresponding profiles $V^{1}, \ldots, V^{q}$.

- Step 2. Replicate the previous profiles, taking into account the corresponding percentages $100 \cdot w_{1}, \ldots, 100 \cdot w_{q}$. In practice, calculate the greatest common divisor (gcd) of percentages associated with the weights, and divide each percentage by the gcd. Then, the minimum number of replications of each profile is obtained:

$$
t_{i}=\frac{100 \cdot w_{i}}{\operatorname{gcd}\left\{100 \cdot w_{1}, \ldots, 100 \cdot w_{q}\right\}}, \quad i \in\{1, \ldots, q\} .
$$

- Step 3. For each alternative $x_{i} \in X$, calculate the ordinal proximities between the obtained assessments (taking into account the corresponding replications) and $l_{g}$ :

$$
\pi^{*}\left(v_{i}^{1,1}, l_{g}\right), \ldots, \pi^{*}\left(v_{i}^{m, 1}, l_{g}\right), \ldots, \pi^{*}\left(v_{i}^{1, q}, l_{g}\right), \ldots, \pi^{*}\left(v_{i}^{m, q}, l_{g}\right) \in \Delta_{2} .
$$

- Step 4. For each alternative $x_{i} \in X$, arrange the previous pairs of ordinal degrees of proximity in a decreasing fashion with respect to the linear order $\succeq_{2}$ on $\Delta_{2}$.

- Step 5. For each alternative $x_{i} \in X$, select the medians $M_{i} \in \Delta_{4}$, with

$$
\Delta_{4}=\left\{\left(\left(\delta_{r}, \delta_{s}\right),\left(\delta_{t}, \delta_{u}\right)\right) \in\left(\Delta_{2}\right)^{2} \mid\left(\delta_{r}, \delta_{s}\right) \succeq_{2}\left(\delta_{t}, \delta_{u}\right)\right\},
$$

in the following way:

1. If the number of pairs of ordinal degrees of proximity is even, then consider the two medians. For instance,

$$
\left(\left(\delta_{1}, \delta_{1}\right),\left(\delta_{1}, \delta_{2}\right),\left(\delta_{2}, \delta_{2}\right),\left(\delta_{2}, \delta_{2}\right)\right) \mapsto\left(\left(\delta_{1}, \delta_{2}\right),\left(\delta_{2}, \delta_{2}\right)\right) \in \Delta_{4}
$$


2. If the number of pairs of ordinal degrees of proximity is odd, then duplicate the median. For instance,

$$
\left(\left(\delta_{1}, \delta_{1}\right),\left(\delta_{1}, \delta_{2}\right),\left(\delta_{2}, \delta_{2}\right)\right) \mapsto\left(\left(\delta_{1}, \delta_{2}\right),\left(\delta_{1}, \delta_{2}\right)\right) \in \Delta_{4}
$$

The proposed procedure uses medians like the Majority Judgment voting system introduced by Balinski and Laraki $[1,2]$. However, while Majority Judgment considers the lower median of individual assessments, in our proposal we select the pair of medians of the degrees of proximity between individual assessments and the highest possible assessment. Therefore, the medians are of different nature. In addition, our procedure avoids the loss of information by considering the two medians when the number of assessments is even.

- Step 6. Rank order the alternatives through the weak order $\succcurlyeq_{4}$ on $\Delta_{4}$, defined as

$$
\begin{aligned}
& \left(\left(\delta_{r}, \delta_{s}\right),\left(\delta_{t}, \delta_{u}\right)\right) \succcurlyeq_{4}\left(\left(\delta_{r^{\prime}}, \delta_{s^{\prime}}\right),\left(\delta_{t^{\prime}}, \delta_{u^{\prime}}\right)\right) \Leftrightarrow \\
& \left\{\begin{array}{l}
r+s+t+u<r^{\prime}+s^{\prime}+t^{\prime}+u^{\prime} \\
\text { or } \\
r+s+t+u=r^{\prime}+s^{\prime}+t^{\prime}+u^{\prime} \text { and } \\
D\left(\delta_{r}, \delta_{s}, \delta_{t}, \delta_{u}\right) \leq D\left(\delta_{r^{\prime}}, \delta_{s^{\prime}}, \delta_{t^{\prime}}, \delta_{u^{\prime}}\right),
\end{array}\right.
\end{aligned}
$$

where $D\left(\delta_{r}, \delta_{s}, \delta_{t}, \delta_{u}\right)=|r-s|+|r-t|+|r-u|+|s-t|+|s-u|+|t-u|$ measures the dispersion in $\left(\delta_{r}, \delta_{s}, \delta_{t}, \delta_{u}\right)$.

Notice that $\succeq_{4}$ follows the same pattern that $\succeq_{2}$ : first focus on the sum of the subindices and then on the dispersion $\left(D\left(\delta_{r}, \delta_{s}\right)=|r-s|=s-r\right)$. However, $\succcurlyeq_{4}$ is not a linear order. For instance, $\left(\left(\delta_{1}, \delta_{3}\right),\left(\delta_{2}, \delta_{3}\right)\right)$ and $\left(\left(\delta_{1}, \delta_{2}\right),\left(\delta_{3}, \delta_{3}\right)\right)$ are indifferent: $1+3+2+3=1+2+3+3=9$ and $D\left(\delta_{1}, \delta_{3}, \delta_{2}, \delta_{3}\right)=D\left(\delta_{1}, \delta_{2}, \delta_{3}, \delta_{3}\right)=7$.

Then, the weak order $\succcurlyeq X$ on $X$ is defined as

$$
x_{i} \succcurlyeq_{X} x_{j} \Leftrightarrow M_{i} \succcurlyeq_{4} M_{j},
$$

for all $x_{i}, x_{j} \in X$.

Notice that the median operator is invariant under replications. Thus, if 
in Step 2 (see Eq. (1)) the replications are $k \cdot t_{i}$, the medians are the same, for every positive integer $k$.

- Step \%. Since some alternatives can be indifferent with respect to $\succcurlyeq_{4}$, it is necessary to devise a tie-breaking process for ordering the alternatives. We propose to use a sequential procedure based on Balinski and Laraki [1] (see Balinski and Laraki [3] for practical examples). It consists of withdrawing the medians of the alternatives that are in a tie, and then selecting the new medians for the corresponding alternatives and applying steps 5 and 6 . The process continues until the ties are broken.

\section{Case study}

In this section, we apply the proposed MCDM procedure for ranking wines during a blind wine tasting carried out in Consejo Regulador de la Denominación de Origen Cigales (Cigales, Valladolid, Spain, October 10th, 2017), under appropriate conditions of temperature, light and service, without communication among experts.

Nine wine experts recruited by Academia Castellano Leonesa de Gastronomía y Alimentación tasted eight red wines from Cigales Protected Designation of Origin using the five linguistic terms of Table 1 (or the corresponding interval of two consecutive linguistic terms, when they hesitated). The wines' assessments made by each expert are included in the Appendix.

The nine experts assessed each wine taking into account the most important sensory aspects in wine tasting: appearance, smell and taste, as well as essential factors of each criterion, such as clarity, color, intensity and quality, and also the overall impression (see Jackson [17]).

Table 2 contains the frequencies and percentages of the assessments in global terms. Notice that the proportions of $l_{3}$ and $l_{4}$ reached $69.6 \%$ of the assessments, while $l_{1}$ and $\left[l_{1}, l_{2}\right]$ only represented $0.4 \%$. Also, it is important to remark that eight out the nine experts used sometimes two consecutive terms, being this type of the assessments 64 out of 504, i.e., approximately $12.7 \%$ of the cases.

Most tasting sheets, like Davis 20-point scorecard, use detailed score sheets for evaluating sensory aspects of wines, giving a particular weight to each aspect in the total score (see Jackson [17]). Since each sensory aspect may have different importance in wine tasting, a weight was assigned to each criterion ${ }^{5}$. Aspects,

${ }^{5}$ These weights were obtained from the usual tasting sheet used by the Consejo Regulador 


\begin{tabular}{ll}
\hline$l_{1}$ & Insufficient \\
$l_{2}$ & Fair \\
$l_{3}$ & Good \\
$l_{4}$ & Very good \\
$l_{5}$ & Excellent \\
\hline
\end{tabular}

Table 1: Linguistic terms in $\mathcal{L}$.

\begin{tabular}{ccc} 
& Frequency & Percentages $(\%)$ \\
\hline$l_{1}$ & 1 & 0.2 \\
{$\left[l_{1}, l_{2}\right]$} & 1 & 0.2 \\
$l_{2}$ & 43 & 8.5 \\
{$\left[l_{2}, l_{3}\right]$} & 14 & 2.8 \\
$l_{3}$ & 178 & 35.3 \\
{$\left[l_{3}, l_{4}\right]$} & 34 & 6.7 \\
$l_{4}$ & 173 & 34.3 \\
{$\left[l_{4}, l_{5}\right]$} & 15 & 3.0 \\
$l_{5}$ & 45 & 8.9 \\
\hline & 504 & 100
\end{tabular}

Table 2: Summary of the assessments given by the wine experts.

weights and the corresponding replications considered in the case study are shown in Table 3.

In order to know if the OQS used in the wine tasting was perceived as uniform, after the wine tasting we asked the nine wine experts to complete an on-line survey regarding the proximities between the terms of the OQS of Table 1.

With the information obtained in the survey, we generated the metrizable OPM for each expert following the algorithm introduced in García-Lapresta et al. $[9,2.3]$. This algorithm starts by asking the expert about the comparison between the ordinal proximities $\pi_{12}$ and $\pi_{23}$. The next question differs depending on whether one of these ordinal degrees of proximity is greater than the other or they are the same. The algorithm continues with similar questions

de la Denominación de Origen Cigales. 


\begin{tabular}{llrl}
\hline Appearance & & $15 \%$ & \\
& Clarity & $5 \%$ & \\
& Color & $10 \%$ & 2 replications \\
& & & \\
\hline \multirow{2}{*}{ Smell } & & $30 \%$ & \\
& Intensity & $10 \%$ & 2 replications \\
& Quality & $20 \%$ & 4 replications \\
\hline \multirow{2}{*}{ Taste } & & $45 \%$ & \\
& Intensity & $10 \%$ & 2 replications \\
& Quality & $35 \%$ & 7 replications \\
\hline Overall impression & & $10 \%$ & 2 replications \\
\hline
\end{tabular}

Table 3: Sensory aspects and replications.

comparing the ordinal proximities between the remaining pairs of terms of the OQS until the metrizable OPM is obtained.

The matrices associated with the metrizable OPMs of the nine experts were

$$
\begin{aligned}
A_{3223}= & \left(\begin{array}{lllll}
\delta_{1} & \delta_{3} & \delta_{5} & \delta_{6} & \delta_{7} \\
& \delta_{1} & \delta_{2} & \delta_{4} & \delta_{6} \\
& & \delta_{1} & \delta_{2} & \delta_{5} \\
& & & \delta_{1} & \delta_{3} \\
& & & & \delta_{1}
\end{array}\right), A_{3234}
\end{aligned}
$$




$$
A_{3432}^{\prime}=\left(\begin{array}{ccccc}
\delta_{1} & \delta_{3} & \delta_{6} & \delta_{8} & \delta_{9} \\
& \delta_{1} & \delta_{4} & \delta_{6} & \delta_{7} \\
& & \delta_{1} & \delta_{3} & \delta_{5} \\
& & & \delta_{1} & \delta_{2} \\
& & & & \delta_{1}
\end{array}\right) .
$$

The frequency of these matrices is shown in Table 4. It is interesting to note that none of the experts perceived the OQS as uniform.

\begin{tabular}{ccc} 
Matrix & Frequency & $\%$ \\
\hline$A_{3223}$ & 1 & 11.11 \\
$A_{3234}$ & 1 & 11.11 \\
$A_{3342}$ & 2 & 22.22 \\
$A_{3432}$ & 4 & 44.44 \\
$A_{3432}^{\prime}$ & 1 & 11.11 \\
\hline & 9 & 100
\end{tabular}

Table 4: Matrices of the survey.

Since experts' opinions about the proximities between the linguistic terms of the OQS produced 5 different metrizable OPMs, it was advisable to find a collective metrizable OPM that represents individual opinions as faithfully as possible. To do that, we applied the aggregation procedure introduced in García-Lapresta et al. [9, 4.3]. The procedure is based on weighted-metrics and it provides the metrizable OPM that minimizes the sum of distances (square distances) between itself and the metrizable OPMs of the experts.

The final outcome obtained after applying the mentioned aggregation procedure was the metrizable OPM associated with the proximity matrix $A_{3432}$ that can be visualized in Fig. 2 .

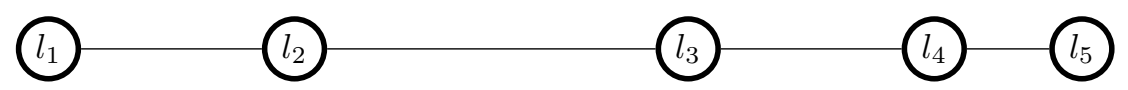

Figure 2: Metrizable OPM with associated matrix $A_{3432}$.

Once the metrizable OPM was obtained, we generated the extension of $\pi$ to $\mathcal{L}_{2}$. As we have seen in Section 3, the proposed ordinal MCDM procedure 
considers the proximities between the assessments given by agents and $l_{g}$. Thus, we considered the pairs of $\delta$ 's contained in the last column of the proximity matrix associated with $\pi^{*}$ :

$$
\left(\begin{array}{c}
\pi^{*}\left(l_{1}, l_{5}\right) \\
\pi^{*}\left(\left[l_{1}, l_{2}\right], l_{5}\right) \\
\pi^{*}\left(l_{2}, l_{5}\right) \\
\pi^{*}\left(\left[l_{2}, l_{3}\right], l_{5}\right) \\
\pi^{*}\left(l_{3}, l_{5}\right) \\
\pi^{*}\left(\left[l_{3}, l_{4}\right], l_{5}\right) \\
\pi^{*}\left(l_{4}, l_{5}\right) \\
\pi^{*}\left(\left[l_{4}, l_{5}\right], l_{5}\right) \\
\pi^{*}\left(l_{5}, l_{5}\right)
\end{array}\right)=\left(\begin{array}{c}
\left(\delta_{8}, \delta_{8}\right) \\
\left(\delta_{6}, \delta_{8}\right) \\
\left(\delta_{6}, \delta_{6}\right) \\
\left(\delta_{4}, \delta_{6}\right) \\
\left(\delta_{4}, \delta_{4}\right) \\
\left(\delta_{2}, \delta_{4}\right) \\
\left(\delta_{2}, \delta_{2}\right) \\
\left(\delta_{1}, \delta_{2}\right) \\
\left(\delta_{1}, \delta_{1}\right)
\end{array}\right) .
$$

After replicating the profiles according to Table 3 and applying the steps 3 , 4,5 and 6 , we obtain the following pairs of medians:

$$
\begin{gathered}
M_{1}=M_{3}=M_{5}=M_{6}=M_{7}=\left(\left(\delta_{4}, \delta_{4}\right),\left(\delta_{4}, \delta_{4}\right)\right), \\
M_{2}=M_{8}=\left(\left(\delta_{2}, \delta_{4}\right),\left(\delta_{2}, \delta_{4}\right)\right), \quad M_{4}=\left(\left(\delta_{2}, \delta_{2}\right),\left(\delta_{2}, \delta_{2}\right)\right) .
\end{gathered}
$$

Then, $M_{4} \succ_{4}\left(M_{2} \sim_{4} M_{8}\right) \succ_{4}\left(M_{1} \sim_{4} M_{3} \sim_{4} M_{5} \sim_{4} M_{6} \sim_{4} M_{7}\right)$.

In order to break the ties between wines 2 and 8 , and wines 1, 3, 5, 6 and 7 , it is necessary to use the tie-breaking procedure of step 7 .

For wines 2 and 8 , after two tiebreakers, we have:

$$
M_{2}=\left(\left(\delta_{2}, \delta_{4}\right),\left(\delta_{4}, \delta_{4}\right)\right), \quad M_{8}=\left(\left(\delta_{2}, \delta_{4}\right),\left(\delta_{2}, \delta_{4}\right)\right)
$$

Then, $M_{4} \succ_{4} M_{8} \succ_{4} M_{2} \succ_{4}\left(\begin{array}{lllllllll}M_{1} \sim_{4} & M_{3} \sim_{4} & M_{5} \sim_{4} & M_{6} \sim_{4} & M_{7}\end{array}\right)$.

For wines $1,3,5,6$ and 7 , after applying a tiebreaker, we have:

$$
M_{1}=\left(\left(\delta_{2}, \delta_{4}\right),\left(\delta_{4}, \delta_{4}\right)\right), \quad M_{3}=M_{5}=M_{6}=M_{7}=\left(\left(\delta_{4}, \delta_{4}\right),\left(\delta_{4}, \delta_{4}\right)\right) .
$$

Then, $M_{4} \succ_{4} M_{8} \succ_{4} M_{2} \succ_{4} M_{1} \succ_{4}\left(\begin{array}{lllll}M_{5} \sim_{4} & M_{3} \sim_{4} & M_{6} \sim_{4} & M_{7}\end{array}\right)$.

After applying again the tie-breaking procedure for the rest of wines, we finally obtain the following ranking:

$$
M_{4} \succ_{4} M_{8} \succ_{4} M_{2} \succ_{4} M_{1} \succ_{4} M_{5} \succ_{4} M_{3} \succ_{4} M_{6} \succ_{4} M_{7} .
$$

Table 5 contains the final ranking of wines and the global assessment obtained for each wine expressed in linguistic terms. These assessments have been 


\begin{tabular}{ccc} 
Position & Wine & Global assessment \\
\hline 1 & 4 & Very good \\
2 & 8 & Good - Very good \\
3 & 2 & Good - Very good \\
4 & 1 & Good \\
5 & 5 & Good \\
6 & 3 & Good \\
7 & 6 & Good \\
8 & 7 & Good
\end{tabular}

Table 5: Ranking of wines obtained after applying the MCDM procedure.

obtained taking into account the initial pairs of ordinal degrees of proximity for each wine and the last column of the proximity matrix associated with $\pi^{*}$ (Eq. $(2))$.

For instance, for wine 2 the initial pair of medians is $M_{2}=\left(\left(\delta_{2}, \delta_{4}\right),\left(\delta_{2}, \delta_{4}\right)\right)$, which correspond to $\left[l_{3}, l_{4}\right]$ in $\pi^{*}$. Thus, the global assessment for the wine 2 is between Good and Very good.

\section{Concluding remarks}

Since words are more appropriate than numbers for expressing human subjective opinions, many decision-making problems use ordered qualitative scales formed by linguistic terms for evaluating alternatives.

In general, the use of ordered qualitative scales is based on the implicit assumption that scales are uniform, assigning a numerical value to each linguistic term of the scale. However, sometimes agents can perceive that the proximities between the terms of the scale are not identical. In these cases, the conversion of linguistic terms into numerical values is meaningless, being more appropriate an ordinal approach for dealing with these non-uniform ordered qualitative scales.

In this paper, we have introduced a MCDM procedure that is based on the ordinal proximities between the highest linguistic term of the scale and the individual assessments (a single term or two consecutive linguistic terms of the scale, when they hesitate).

It is worth pointing out that our proposal avoids the use of numerical values and follows an ordinal approach, in such a way that the weights assigned to 
criteria are used in a qualitative fashion by replicating experts' opinions proportionally to the weights.

Section 4 illustrated the practicality of the procedure in a wine tasting where experts assessed eight wines regarding different criteria. However, our procedure can be implemented in many different scenarios such as satisfaction surveys, quality of life questionnaires, or quality controls in manufacturing processes, when agents evaluate a set of alternatives using the same ordered qualitative scale for each criterion.

For further research, it could be interesting to devise a MCDM procedure that considers different ordered qualitative scales (equipped with the corresponding ordinal proximity measures) associated with the criteria.

\section{Acknowledgments.}

The authors are grateful to Academia Castellano Leonesa de Gastronomía y Alimentación and Consejo Regulador de la Denominación de Origen Cigales, specially to his president, Julio Valles, to the nine wine experts for their collaboration in the wine tasting, and also to three anonymous referees for their useful comments and suggestions. The financial support of the Spanish Ministerio de Economía y Competitividad (project ECO2016-77900-P) and ERDF is acknowledged.

\section{References}

[1] Balinski, M., Laraki, R.: A theory of measuring, electing and ranking. Proceedings of the National Academy of Sciences of the United States of America 104, pp. 8720-8725, 2007.

[2] Balinski, M., Laraki, R.: Majority Judgment: Measuring, Ranking, and Electing. The MIT Press, Cambridge, MA, 2011.

[3] Balinski, M., Laraki, R.: How best to rank wines: Majority Judgment, in: Wine Economics: Quantitative Studies and Empirical Observations. Palgrave-MacMillan, pp. 149-172, 2013.

[4] Bustince, H., Fernández, J., Kolesárová, A., Mesiar, R.: Generation of linear orders for intervals by means of aggregation functions. Fuzzy Sets and Systems 220, pp. 69-77, 2013. 
[5] Falcó, E., García-Lapresta, J.L., Roselló, L.: Allowing agents to be imprecise: A proposal using multiple linguistic terms. Information Sciences 258, pp. 249-265, 2014.

[6] Fasolo, B., Bana e Costa, C.A.: Tailoring value elicitation to decision makers' numeracy and fluency: Expressing value judgments in numbers or words. Omega 44, pp. 83-90, 2014.

[7] Franceschini, F. , Galetto, M., Varetto, M.: Qualitative ordinal scales: the concept of ordinal range. Quality Engineering 16, pp. 515-524, 2004.

[8] García-Lapresta, J.L., González del Pozo, R.: An ordinal multi-criteria decision-making procedure in the context of uniform qualitative scales, in: Soft Computing Applications for Group Decision-making and Consensus Modeling (eds. M. Collan, J. Kacprzyk). Studies in Fuzziness and Soft Computing 357. Springer, pp. 297-304, 2018.

[9] García-Lapresta, J.L., González del Pozo, R., Pérez-Román, D.: Metrizable ordinal proximity measures and their aggregation. Information Sciences 448-449, pp. 149-163, 2018.

[10] García-Lapresta, J.L., Pérez-Román, D.: Ordinal proximity measures in the context of unbalanced qualitative scales and some applications to consensus and clustering. Applied Soft Computing 35, pp. 864-872, 2015.

[11] García-Lapresta, J.L., Pérez-Román, D.: A consensus reaching process in the context of non-uniform ordered qualitative scales. Fuzzy Optimization and Decision Making 16, pp. 449-461, 2017.

[12] García-Lapresta, J.L., Pérez-Román, D.: Aggregating opinions in nonuniform ordered qualitative scales. Applied Soft Computing 67, pp. 652$657,2018$.

[13] Greco, S., Ehrgott, M., Figueira, J.R. (Eds.): Multiple Criteria Decision Analysis: State of the Art Surveys. International Series in Operations Research \& Management Science 233, Springer, 2016.

[14] Herrera, F., Herrera-Viedma, E., Martínez, L.: A fuzzy linguistic methodology to deal with unbalanced linguistic term sets. IEEE Transactions on Fuzzy Systems 16, pp. 354-370, 2008. 
[15] Herrera-Viedma, E., López-Herrera, A.G.: A model of an information retrieval system with unbalanced fuzzy linguistic information. International Journal of Intelligent Systems 22, pp. 1197-1214, 2007.

[16] Hong-Bin, Y., Tieju, M., Van-Nam, H.: On qualitative multi-attribute group decision making and its consensus measure: A probability based perspective. Omega 70, pp. 94-117, 2017.

[17] Jackson, R.S.: Wine Tasting: A Professional Handbook. Elsevier Academic Press, 2017.

[18] Kolesárová, A., Mayor, G., Mesiar, R.: Weighted ordinal means. Information Sciences 177, pp. 3822-3830, 2007.

[19] Larichev, O., Brown, R.: Numerical and verbal decision analysis: comparison on practical cases. Journal of Multi-Criteria Decision Analysis 9, pp. 263-274, 2000.

[20] Likert, R.: A technique for the measurement of attitudes. Archives of Psychology 22 (140), pp. 1-55, 1932.

[21] Lozano, L.M, García-Cueto, E., Muñiz, J.: Effect of the number of response categories on the reliability and validity of rating scales. European Journal of Research Methods for the Behavioral and Social Sciences 4, pp. 73-79, 2008.

[22] Miller, G.A.: The magical number seven, plus or minus two: some limits on our capacity for processing information. Psychological Review, 63(2), pp. 81-97, 1956.

[23] Roberts, F.S.: Measurement Theory. Addison-Wesley Publishing Company, Reading, 1979.

[24] Teigen, K.H.: The language of uncertainty. Acta Psychologica 68, pp. 2738, 1988.

[25] Travé-Massuyès, L., Piera, N.: The orders of magnitude models as qualitative algebras. Proceedings of the 11th International Joint Conference on Artificial Intelligence 2, pp. 1261-1266, 1989.

[26] Windschitl, P.D., Wells, G.L.: Measuring psychological uncertainty: Verbal versus numeric methods. Journal of Experimental Psychology: Applied 2, pp. 343-364, 1996. 
[27] Xu, Z.S., Yager, R.R.: Some geometric aggregation operators based on intuitionistic fuzzy sets. International Journal of General Systems 8, pp. 417-433, 2006.

[28] Zadeh, L.A.: The concept of a linguistic variable and its application to approximate reasoning. Information Sciences 8, pp. 301-357, 1978.

[29] Zimmer, A.C.: Verbal vs. numerical processing of subjective probabilities. Decision Making Under Uncertainty 16, pp. 159-182, 1983. 


\section{Appendix}

\begin{tabular}{ccccccccccc}
\hline & & 1 & 2 & 3 & 4 & 5 & 6 & 7 & 8 & 9 \\
\hline \multirow{2}{*}{ Appearance } & Clarity & $l_{4}$ & $l_{4}$ & $l_{5}$ & $l_{3}$ & $l_{3}$ & $l_{4}$ & $l_{3}$ & $l_{4}$ & $l_{3}$ \\
& Color & $l_{4}$ & $l_{4}$ & $l_{4}$ & $l_{4}$ & $l_{3}$ & $l_{4}$ & $l_{3}$ & {$\left[l_{3}, l_{4}\right]$} & {$\left[l_{3}, l_{4}\right]$} \\
\hline \multirow{2}{*}{ Smell } & Intensity & $l_{4}$ & $l_{5}$ & $l_{4}$ & $l_{3}$ & $l_{3}$ & $l_{3}$ & $l_{4}$ & {$\left[l_{3}, l_{4}\right]$} & {$\left[l_{3}, l_{4}\right]$} \\
& Quality & $l_{4}$ & $l_{4}$ & $l_{4}$ & $l_{3}$ & $l_{3}$ & {$\left[l_{3}, l_{4}\right]$} & $l_{3}$ & {$\left[l_{3}, l_{4}\right]$} & $l_{4}$ \\
\hline \multirow{2}{*}{ Taste } & Intensity & $l_{4}$ & $l_{4}$ & $l_{4}$ & $l_{3}$ & $l_{2}$ & $l_{4}$ & $l_{3}$ & $l_{3}$ & {$\left[l_{3}, l_{4}\right]$} \\
& Quality & $l_{3}$ & $l_{3}$ & $l_{3}$ & $l_{3}$ & $l_{2}$ & $l_{4}$ & $l_{3}$ & $l_{3}$ & $l_{4}$ \\
\hline \multicolumn{2}{c}{ Overall impression } & $l_{4}$ & $l_{4}$ & {$\left[l_{3}, l_{4}\right]$} & $l_{3}$ & {$\left[l_{2}, l_{3}\right]$} & {$\left[l_{3}, l_{4}\right]$} & $l_{3}$ & $l_{3}$ & {$\left[l_{3}, l_{4}\right]$} \\
\hline
\end{tabular}

Table 6: Assessments obtained for wine 1.

\begin{tabular}{ccccccccccc}
\hline & & 1 & 2 & 3 & 4 & 5 & 6 & 7 & 8 & 9 \\
\hline \multirow{2}{*}{ Appearance } & Clarity & $l_{5}$ & $l_{4}$ & $l_{5}$ & $l_{5}$ & $l_{3}$ & $l_{4}$ & $l_{3}$ & $l_{4}$ & $l_{4}$ \\
& Color & $l_{4}$ & $l_{4}$ & $l_{4}$ & $l_{4}$ & $l_{4}$ & $l_{2}$ & $l_{3}$ & $l_{3}$ & $l_{4}$ \\
\hline \multirow{2}{*}{ Smell } & Intensity & $l_{4}$ & $l_{4}$ & $l_{3}$ & $l_{4}$ & {$\left[l_{2}, l_{3}\right]$} & $l_{3}$ & $l_{4}$ & {$\left[l_{3}, l_{4}\right]$} & $l_{3}$ \\
& Quality & {$\left[l_{4}, l_{5}\right]$} & $l_{3}$ & $l_{4}$ & $l_{4}$ & $l_{3}$ & $l_{3}$ & $l_{3}$ & $l_{3}$ & $l_{3}$ \\
\hline \multirow{2}{*}{ Taste } & Intensity & $l_{4}$ & {$\left[l_{3}, l_{4}\right]$} & $l_{4}$ & $l_{3}$ & $l_{3}$ & $l_{3}$ & $l_{2}$ & $l_{4}$ & $l_{3}$ \\
& Quality & $l_{4}$ & {$\left[l_{3}, l_{4}\right]$} & $l_{4}$ & $l_{3}$ & $l_{2}$ & $l_{2}$ & $l_{2}$ & $l_{4}$ & $l_{4}$ \\
\hline \multicolumn{2}{c}{ Overall impression } & $l_{4}$ & $l_{3}$ & {$\left[l_{3}, l_{4}\right]$} & $l_{4}$ & $l_{2}$ & $l_{3}$ & {$\left[l_{2}, l_{3}\right]$} & {$\left[l_{3}, l_{4}\right]$} & $l_{3}$ \\
\hline
\end{tabular}

Table 7: Assessments obtained for wine 2. 


\begin{tabular}{ccccccccccc}
\hline & & 1 & 2 & 3 & 4 & 5 & 6 & 7 & 8 & 9 \\
\hline \multirow{2}{*}{ Appearance } & Clarity & $l_{5}$ & $l_{4}$ & $l_{4}$ & $l_{5}$ & $l_{4}$ & $l_{4}$ & $l_{4}$ & $l_{4}$ & $l_{3}$ \\
& Color & $l_{5}$ & $l_{4}$ & $l_{3}$ & $l_{5}$ & $l_{4}$ & $l_{3}$ & $l_{4}$ & $l_{4}$ & $l_{4}$ \\
\hline \multirow{2}{*}{ Smell } & Intensity & $l_{4}$ & $l_{4}$ & $l_{4}$ & $l_{3}$ & $l_{4}$ & $l_{3}$ & $l_{4}$ & $l_{4}$ & $l_{4}$ \\
& Quality & $l_{2}$ & $l_{2}$ & $l_{3}$ & $l_{3}$ & $l_{3}$ & $l_{2}$ & $l_{3}$ & $l_{4}$ & {$\left[l_{3}, l_{4}\right]$} \\
\hline \multirow{2}{*}{ Taste } & Intensity & $l_{2}$ & $l_{3}$ & $l_{4}$ & $l_{3}$ & $l_{3}$ & $l_{3}$ & $l_{4}$ & $l_{4}$ & $l_{4}$ \\
& Quality & $l_{1}$ & $l_{2}$ & $l_{3}$ & $l_{4}$ & $l_{3}$ & {$\left[l_{2}, l_{3}\right]$} & $l_{3}$ & $l_{4}$ & {$\left[l_{3}, l_{4}\right]$} \\
\hline \multicolumn{2}{c}{ Overall impression } & $l_{2}$ & $l_{2}$ & $l_{3}$ & $l_{4}$ & {$\left[l_{3}, l_{4}\right]$} & {$\left[l_{2}, l_{3}\right]$} & {$\left[l_{3}, l_{4}\right]$} & $l_{4}$ & {$\left[l_{3}, l_{4}\right]$} \\
\hline
\end{tabular}

Table 8: Assessments obtained for wine 3.

\begin{tabular}{ccccccccccc}
\hline & & 1 & 2 & 3 & 4 & 5 & 6 & 7 & 8 & 9 \\
\hline \multirow{2}{*}{ Appearance } & Clarity & $l_{5}$ & $l_{4}$ & $l_{5}$ & $l_{4}$ & $l_{4}$ & $l_{4}$ & $l_{4}$ & $l_{4}$ & {$\left[l_{3}, l_{4}\right]$} \\
& Color & $l_{5}$ & $l_{5}$ & $l_{4}$ & $l_{5}$ & $l_{4}$ & $l_{4}$ & $l_{4}$ & $l_{4}$ & {$\left[l_{3}, l_{4}\right]$} \\
\hline \multirow{2}{*}{ Smell } & Intensity & $l_{5}$ & $l_{5}$ & $l_{4}$ & $l_{5}$ & $l_{4}$ & $l_{5}$ & $l_{3}$ & {$\left[l_{4}, l_{5}\right]$} & {$\left[l_{3}, l_{4}\right]$} \\
& Quality & $l_{5}$ & $l_{4}$ & $l_{3}$ & $l_{4}$ & $l_{3}$ & $l_{5}$ & $l_{3}$ & $l_{4}$ & $l_{3}$ \\
\hline \multirow{2}{*}{ Taste } & Intensity & $l_{5}$ & $l_{4}$ & $l_{4}$ & $l_{5}$ & $l_{3}$ & $l_{4}$ & $l_{3}$ & $l_{4}$ & $l_{4}$ \\
& Quality & $l_{5}$ & $l_{3}$ & {$\left[l_{3}, l_{4}\right]$} & $l_{4}$ & $l_{3}$ & $l_{4}$ & $l_{3}$ & {$\left[l_{3}, l_{4}\right]$} & {$\left[l_{4}, l_{5}\right]$} \\
\hline \multicolumn{2}{c}{ Overall impression } & $l_{5}$ & $l_{4}$ & $l_{4}$ & $l_{5}$ & $l_{3}$ & $l_{4}$ & $l_{3}$ & $l_{4}$ & $l_{4}$ \\
\hline
\end{tabular}

Table 9: Assessments obtained for wine 4.

\begin{tabular}{ccccccccccc}
\hline & & 1 & 2 & 3 & 4 & 5 & 6 & 7 & 8 & 9 \\
\hline \multirow{2}{*}{ Appearance } & Clarity & $l_{5}$ & $l_{4}$ & $l_{4}$ & $l_{3}$ & $l_{4}$ & {$\left[l_{3}, l_{4}\right]$} & $l_{4}$ & $l_{4}$ & {$\left[l_{4}, l_{5}\right]$} \\
& Color & $l_{5}$ & $l_{5}$ & $l_{4}$ & $l_{3}$ & $l_{4}$ & $l_{3}$ & $l_{4}$ & $l_{4}$ & $l_{4}$ \\
\hline \multirow{2}{*}{ Smell } & Intensity & $l_{3}$ & $l_{5}$ & $l_{3}$ & $l_{3}$ & $l_{4}$ & $l_{3}$ & $l_{4}$ & $l_{4}$ & $l_{3}$ \\
& Quality & $l_{3}$ & $l_{5}$ & $l_{2}$ & $l_{3}$ & $l_{3}$ & $l_{4}$ & $l_{3}$ & {$\left[l_{3}, l_{4}\right]$} & {$\left[l_{3}, l_{4}\right]$} \\
\hline \multirow{2}{*}{ Taste } & Intensity & $l_{4}$ & $l_{4}$ & $l_{4}$ & $l_{2}$ & $l_{3}$ & $l_{3}$ & $l_{2}$ & $l_{4}$ & $l_{4}$ \\
& Quality & $l_{3}$ & {$\left[l_{4}, l_{5}\right]$} & $l_{3}$ & $l_{2}$ & $l_{2}$ & $l_{3}$ & $l_{3}$ & $l_{4}$ & $l_{4}$ \\
\hline \multicolumn{2}{c}{ Overall impression } & $l_{3}$ & {$\left[l_{4}, l_{5}\right]$} & $l_{3}$ & $l_{3}$ & $l_{3}$ & $l_{3}$ & {$\left[l_{3}, l_{4}\right]$} & $l_{4}$ & $l_{4}$ \\
\hline
\end{tabular}

Table 10: Assessments obtained for wine 5.

\begin{tabular}{ccccccccccc}
\hline & & 1 & 2 & 3 & 4 & 5 & 6 & 7 & 8 & 9 \\
\hline \multirow{2}{*}{ Appearance } & Clarity & $l_{5}$ & $l_{4}$ & $l_{5}$ & $l_{4}$ & $l_{4}$ & $l_{3}$ & $l_{3}$ & $l_{4}$ & {$\left[l_{4}, l_{5}\right]$} \\
& Color & $l_{5}$ & {$\left[l_{2}, l_{3}\right]$} & $l_{3}$ & $l_{3}$ & $l_{4}$ & $l_{3}$ & $l_{3}$ & $l_{3}$ & {$\left[l_{3}, l_{4}\right]$} \\
\hline \multirow{2}{*}{ Smell } & Intensity & $l_{3}$ & $l_{3}$ & $l_{3}$ & $l_{2}$ & $l_{3}$ & $l_{4}$ & $l_{4}$ & $l_{3}$ & $l_{3}$ \\
& Quality & $l_{2}$ & $l_{4}$ & $l_{3}$ & $l_{3}$ & $l_{2}$ & $l_{4}$ & $l_{3}$ & $l_{3}$ & $l_{3}$ \\
\hline \multirow{2}{*}{ Taste } & Intensity & $l_{3}$ & $l_{3}$ & $l_{3}$ & $l_{4}$ & $l_{3}$ & $l_{4}$ & $l_{2}$ & $l_{3}$ & $l_{3}$ \\
& Quality & $l_{3}$ & $l_{2}$ & $l_{3}$ & $l_{3}$ & $l_{3}$ & $l_{3}$ & $l_{3}$ & $l_{3}$ & $l_{3}$ \\
\hline \multicolumn{2}{c}{ Overall impression } & $l_{3}$ & $l_{2}$ & $l_{3}$ & $l_{3}$ & $l_{3}$ & {$\left[l_{3}, l_{4}\right]$} & $l_{3}$ & $l_{3}$ & $l_{3}$ \\
\hline
\end{tabular}

Table 11: Assessments obtained for wine 6 . 


\begin{tabular}{ccccccccccc}
\hline & & 1 & 2 & 3 & 4 & 5 & 6 & 7 & 8 & 9 \\
\hline \multirow{2}{*}{ Appearance } & Clarity & $l_{5}$ & $l_{3}$ & $l_{5}$ & $l_{4}$ & $l_{3}$ & $l_{2}$ & $l_{3}$ & $l_{4}$ & $l_{3}$ \\
& Color & $l_{5}$ & $l_{4}$ & $l_{4}$ & $l_{3}$ & $l_{3}$ & $l_{3}$ & $l_{3}$ & $l_{4}$ & $l_{3}$ \\
\hline \multirow{2}{*}{ Smell } & Intensity & $l_{4}$ & {$\left[l_{4}, l_{5}\right]$} & $l_{3}$ & $l_{2}$ & $l_{3}$ & $l_{2}$ & $l_{2}$ & {$\left[l_{4}, l_{5}\right]$} & $l_{4}$ \\
& Quality & $l_{4}$ & $l_{3}$ & $l_{2}$ & $l_{2}$ & $l_{2}$ & $l_{2}$ & $l_{3}$ & {$\left[l_{4}, l_{5}\right]$} & $l_{4}$ \\
\hline \multirow{2}{*}{ Taste } & Intensity & $l_{5}$ & $l_{4}$ & $l_{3}$ & $l_{3}$ & $l_{3}$ & $l_{3}$ & $l_{2}$ & {$\left[l_{4}, l_{5}\right]$} & {$\left[l_{2}, l_{3}\right]$} \\
& Quality & {$\left[l_{4}, l_{5}\right]$} & $l_{3}$ & $l_{3}$ & $l_{3}$ & $l_{2}$ & $l_{2}$ & $l_{2}$ & $l_{4}$ & {$\left[l_{2}, l_{3}\right]$} \\
\hline \multicolumn{2}{c}{ Overall impression } & {$\left[l_{4}, l_{5}\right]$} & $l_{3}$ & {$\left[l_{2}, l_{3}\right]$} & $l_{3}$ & {$\left[l_{2}, l_{3}\right]$} & $l_{2}$ & {$\left[l_{2}, l_{3}\right]$} & $l_{4}$ & {$\left[l_{2}, l_{3}\right]$} \\
\hline
\end{tabular}

Table 12: Assessments obtained for wine 7.

\begin{tabular}{ccccccccccc}
\hline & & 1 & 2 & 3 & 4 & 5 & 6 & 7 & 8 & 9 \\
\hline \multirow{2}{*}{ Appearance } & Clarity & $l_{5}$ & $l_{3}$ & $l_{5}$ & $l_{5}$ & $l_{4}$ & $l_{3}$ & $l_{4}$ & $l_{4}$ & $l_{4}$ \\
& Color & $l_{5}$ & {$\left[l_{4}, l_{5}\right]$} & $l_{3}$ & $l_{5}$ & $l_{4}$ & $l_{3}$ & $l_{3}$ & {$\left[l_{3}, l_{4}\right]$} & {$\left[l_{4}, l_{5}\right]$} \\
\hline \multirow{2}{*}{ Smell } & Intensity & $l_{5}$ & $l_{4}$ & $l_{3}$ & $l_{4}$ & $l_{3}$ & $l_{3}$ & $l_{3}$ & $l_{3}$ & $l_{4}$ \\
& Quality & $l_{4}$ & $l_{4}$ & $l_{3}$ & $l_{5}$ & $l_{3}$ & {$\left[l_{1}, l_{2}\right]$} & $l_{3}$ & $l_{3}$ & {$\left[l_{3}, l_{4}\right]$} \\
\hline \multirow{2}{*}{ Taste } & Intensity & $l_{4}$ & $l_{4}$ & $l_{4}$ & $l_{4}$ & $l_{2}$ & $l_{3}$ & $l_{2}$ & $l_{4}$ & $l_{4}$ \\
& Quality & $l_{4}$ & $l_{3}$ & $l_{3}$ & $l_{4}$ & $l_{2}$ & $l_{3}$ & $l_{2}$ & $l_{4}$ & $l_{4}$ \\
\hline \multicolumn{2}{c}{ Overall impression } & $l_{4}$ & $l_{4}$ & $l_{3}$ & $l_{5}$ & {$\left[l_{2}, l_{3}\right]$} & $l_{3}$ & {$\left[l_{2}, l_{3}\right]$} & {$\left[l_{3}, l_{4}\right]$} & $l_{4}$ \\
\hline
\end{tabular}

Table 13: Assessments obtained for wine 8. 